\title{
A possible molecular mechanism of Chlamydomonas reinhardtii adaptation to different trophic conditions
}

\author{
Roman Puzanskiy ${ }^{1,2}$, Daria Romanyuk ${ }^{3}$, Alexey Shavarda ${ }^{1,2}$, \\ Vladislav Yemelyanov ${ }^{1}$ and Maria Shishova ${ }^{1}$ \\ ${ }^{1}$ Saint Petersburg State University, Saint Petersburg, 199034, Russia \\ ${ }^{2}$ Komarov Botanical Institute of the Russian Academy of Sciences, Saint Petersburg, \\ 197376, Russia \\ ${ }^{3}$ All-Russia Research Institute for Agricultural Microbiology, Saint Petersburg, \\ 196608, Russia
}

| Submitted August 28, 2021| Accepted September 22, 2021 |

\section{Summary}

Microalgae are able to metabolize exogenous organic substrates. Differences in sets of available sources of energy and carbon cause various metabolic and physiological alterations. To characterize those in Chlamydomonas reinhardtii, we compared cells fed with acetate in batch cultures of CC-124 (wt-light) and CC-981 (mutant deficient in phosphoribulokinase, incapable to fix $\mathrm{CO}_{2}$ ), growing under illumination ( $p r k$-light) or in the darkness ( $p r k$-dark). The growth intensity of the $w$ t-light culture was higher only at the beginning of the culture cycle. Its final density only slightly exceeded such of prk-light ones. The growth rate and final density of the prk-dark culture were several times lower.

The GC-MS analysis revealed clear metabolomic differences in cultures with different trophic status. Cells of $p r k$-light were characterized by higher content of fatty acids, sterols, glycolate, malate, and putrescine. Cells of prk-dark accumulated, in particular, relatively higher levels of arginine, ornithine, AMP, some other nitrogencontaining compounds, glycerol, citrate, and sucrose. Higher levels of some amino acids and threonic acid were detected in $w t$-light cells.

Simultaneously, we tested expression of genes encoding key enzymes of plant metabolism. The expression of RBCS1 (Rubisco small subunit), CIS1, 2 (citrate synthase), AAA1 (chloroplast ATP/ADP antiporter), APE2 (triose phosphate translocator), OMT1 (oxoglutarate/malate antiporter) was analyzed. The comparison revealed that $R B C S 1$ was drastically upregulated in prk-light cultures. The expression level of $A A A 1$ in prk-dark cells slightly exceeded such in $w t$-light algae, but was almost zero in prk-light cells. The level of CIS1, 2, APE2 and OMT1 was maximal in $w t$-light cells.

Key words: acetate, batch culture, Chlamydomonas, heterotrophic, metabolomics, mixotrophic, prk1 


\section{Introduction}

For microalgae, light is an important source of energy, and carbon dioxide - of carbon. However, many of them are able to uptake organic substances from the environment to extract energy and/or to assimilate carbon. The variety of potential combinations of trophic types provides microalgae with a wide scope for adaptive strategies. Meanwhile, the mechanisms of combining different types of feeding and their ratio under different conditions remain not completely understood. The study of these mechanisms is not only of great theoretical importance, because it answers the questions of regulation of cell metabolism and its adaptation to changing environmental conditions, but also of practical value, since it can be used for the development of algae biotechnology.

The number of compounds assimilated by many algae includes acetate, and a typical microalga capable of its assimilation is Chlamydomonas reinhardtii. It metabolizes acetate under illumination, maintaining photosynthetic activity, so, it is able to feed mixotrophically. C. reinhardtii can also grow heterotrophically, assimilating acetate in the dark as the only source of energy and carbon (Sager and Granick, 1953). C. reinhardtii is used as a model organism for multiple biological studies (Salomé and Merchant, 2019), such as acclimation to light regime (Mettler et al., 2014), adaptation to mineral nutrition (Grossman, 2000), photosynthetic apparatus structure and functioning (Rochaix, 2001), cell cycle (Cross and Umen, 2015), carbon-concentrating mechanisms (Wang et al., 2015), biofuel studies (Scranton et al., 2015), circadian clock (Matsuo and Ishiura, 2010), flagellar functioning (Ermilova et al., 2000a), chemotaxis responses (Ermilova et al., 1993, 1996, 2000b).

Trophic conditions determine the physiological state of microalgae cells. Depending on the illumination and the presence of the available substrates, there are differences in the cells' growth rate (Sager and Granick, 1953), deposition (Goodson et al., 2011; Moon et al., 2013), respiration intensity (Fett and Coleman, 1994; Terauchi et al., 2010), light (Lucker and Kramer, 2013; Roach et al., 2013) and dark reactions of photosynthesis (Togasaki and Levine, 1970; Heifetz et al., 2000). The power and direction of these changes are determined by trophic specialization, physiological status, type of substrate and many other factors. Thus, under optimal illumination and $\mathrm{CO}_{2}$ content, the addition of a substrate either leads to a decrease in the activity of photosynthesis or does not affect it (Heifetz et al., 2000). However, if conditions limit photosynthesis, its activity increases with the addition of a substrate (Fischer et al., 2006).

The shift of metabolism caused by trophic conditions changing is determined by the activity of biochemical reactions. The latter, in turn, is determined by many factors: modification of already synthesized proteins/enzymes (Lemaire et al., 2004), their degradation (Kajikawa and Fukuzawa, 2020) and/or changes in the expression level of the genes encoding them (Goodenough et al., 2014; Bogaert et al., 2019). In this paper, along with the analysis of the metabolic profiles of $C$. reinhardtii under various trophic conditions, the expression of six genes of key enzymes and transporters involved in carbon metabolism was analyzed.

It is well known that Rubisco plays a key role in carbon fixation. It is a multisubunit enzyme consisting of large and small subunits (Bracher et al., 2017). Small subunits are encoded by nuclear $R B C S$ genes. The presence of organic substances in the environment regulates the intensity of dark photosynthetic reactions in microalgae. Regulation occurs also due to changes in the activity and amount of Rubisco, including that at the transcription level (Hudock and Levine 1964; Togasaki and Levine, 1970; Heifetz et al., 2000; Wienkoop et al., 2010; Puzanskiy et al., 2020a).

In this study, attention will be paid to the enzymes involved in the acetate assimilation. The entry of acetate in the metabolism begins with the formation of a complex with coenzyme A. Then acetyl-CoA can be sent to the tricarboxylic acid cycle (TCA), where the acetate carbon can be oxidized or redirected to gluconeogenesis through the glyoxylate cycle. The entry of acetyl groups into the TCA is carried out by citrate synthase, which catalyzes the reaction of citrate formation from acetyl-CoA and oxaloacetate (Johnson and Alric, 2013). Two genes encoding citrate synthase, CIS1 and CIS2, were identified in the $C$. reinhardtii genome (Atteia et al., 2009). Differences in the localization and functions of these forms are established. It is known that CIS1 is localized in mitochondria and is associated with TCA, and CIS2 participates in the glyoxylate cycle and is located in microbodies (Terashima et al., 2010; Goodenough et al., 2014; Plancke et al., 2014; Lauersen et al., 2016). Since citrate synthases are involved in the utilization of acetate, they play an important role in trophic adaptation, and the 
expression of the genes encoding them depends on the presence of acetate (Wienkoop et al., 2010; Goodenough et al., 2014; Puzanskiy et al., 2020a).

The possibility of importing energy into a plastid is equally important. It has been shown that mutations leading to chloroplast ATPase dysfunction causes the inability of algae to grow under photoautotrophic conditions, but do not take away the ability to grow under heterotrophic conditions (Woessner et al., 1984). However, the dysfunction of ATP synthase in chloroplast can be compensated by the production of ATP by mitochondria (Lemaire et al., 1988). The role of mitochondria in photosynthetic processes and the state of plastids has been demonstrated in a number of works (Bulté et al., 1990; Gans and Rebeille, 1990; Gans and Woolman, 1995; Cardol et al., 2003).

The existence of direct ATP transport mechanism into plastids was shown half a century ago (Heldt, 1969). The mechanism of this transport is associated in many algae with NTT proteins. NTT translocators operate in the mode of exchanging cytoplasmic ATP for stromal ADP (Flugge et al., 2011; Haferkamp et al., 2011; Weber and Linka, 2011; Marchand et al., 2018). The study of NTT properties in higher plants has shown their key role in the physiology of heterotrophic cells (Tjaden et al., 1998; Geigenberger et al., 2001). NTTs play an important role in maintaining plastid metabolism during the dark period (Reiser et al., 2004; Reinhold et al., 2007). Three genes homologous to NTT called $A A A$ were found in the $C$. reinhardtii genome (Johnson and Alric, 2013). Initially, the AAA1 protein was found in the $C$. reinhardtii proteome (Atteia et al., 2009). More recent proteomic studies have shown that in the presence of ethanol, which is toxic to Chlamydomonas, the level of AAA1 increased (Jiang et al., 2017).

Plastid transporters of $\mathrm{C}_{3}$ compounds play an important role in the energy relationship of plastids with cytosol (Ball, 1998). Despite the fact that the genes encoding these transporters were found in the $C$. reinhardtii genome, the proteins were not fully characterized (Johnson and Alric, 2013). Nevertheless, plastid transport of these metabolites has been shown several times (Klein et al., 1983; Klöck and Kreuzberg, 1991; Ball, 1998; Boschetti and Schmid, 1998; Breuers, 2011; Facchinelli and Weber, 2011; Weber and Linka, 2011). It is believed that the transport of these metabolites is handled out by plastid carriers of three-carbon carbohydrates TPT (Heldt and Rapley, 1970; Breuers, 2011; Facchinelli and Weber, 2011; Weber and Linka,
2011). The family of TPT transporters includes the C. reinhardtii protein APE2. These transporters are part of the triosophosphate shuttle, which plays an important role in the balance of reducing power between plastid and cytosol. When the level of $\mathrm{NAD}(\mathrm{P}) \mathrm{H}$ and ATP in the compartment is low, glyceraldehyde-3-phosphate (G3P) will be oxidized to 3-phosphoglycerate (3-PGA) to form NAD $(\mathrm{P}) \mathrm{H}$ and ATP. At high concentrations of $\mathrm{NAD}(\mathrm{P}) \mathrm{H}$ and ATP, the same enzymes catalyze reverse reactions with the absorption of reduced cofactors and ATP. As a result, the cycle, which is balancing the reducing power and ATP between plastid and cytoplasm, is formed (Boschetti and Schmid, 1998; Noguchi and Yoshida, 2008; Johnson and Alric, 2013).

Another mechanism, which regulates redistribution of the reducing power, is malate valve. It allows both to utilize the excess of NADPH in mitochondria and to support the metabolism of chloroplasts by supplying them with reduced cofactors, which is critically important for adapting to changing conditions (Noctor and Foyer, 2000; Scheibe, 2004; Scheibe et al., 2005; Kinoshita et al., 2011). The malate valve is based on the reversible conversion of oxaloacetate to malate by the malate dehydrogenase. With an excess of NADPH in the plastid, oxaloacetate is reduced to malate with the oxidation of NADPH. Malate is then transported to the cytosol by a dicarboxylate transporter, where it is oxidized back to oxaloacetate to form NADH. In the case of a lack of reduced cofactors in the plastid, the process goes in the opposite direction (Scheibe, 2004). The exchange of dicarboxylates between the plastid and the cytosol is carried out by OMT/Dit 1 carriers, which exchange 2-oxoglutarate or oxaloacetate for malate. It has been shown that OMT is responsible for the transport of oxaloacetate into the plastid and is involved in the malate valve (Taniguchi et al., 2002; Kinoshita et al., 2011). The OMT protein was found in the $C$. reinhardtii proteome (Atteia et al., 2009).

The second important OMT function is the involvement in nitrogen exchange. In plant cells, ammonia is assimilated through the plastid cycle of glutamine synthetase/glutamate synthetase (GS/GOGAT). It involves the conversion of 2-oxoglutarate and glutamine into two glutamate molecules (Sanz-Luque et al., 2015). OMT/DiT1 maintains the necessary flow of 2-oxoglutarate into the plastid. DiT2 (glutamate/malate translocator (Facchinelli and Weber, 2011)) is used for the output of glutamate. Antisense suppression of $O M T$ expression leads to the accumulation of 2-oxo- 
glutarate and a decrease in the amino acid content in tobacco (Schneidereit et al., 2006) and arabidopsis (Kinoshita et al., 2011).

The integral function of the metabolism of biological objects is expressed in metabolomics. It is widely used for extended characterization of phenotypes (Weckwerth et al., 2004), studies of adaptive stress-induced reactions (Arbona et al., 2013), and diagnostics of pathological changes (CastroMoretti et al., 2020). The first metabolomic study of $C$. reinhardtii was performed in 2005 (Bölling and Fiehn, 2005). An advanced sample preparation protocol has been developed, which provides rapid and effective "quenching" of metabolism (Lee and Fiehn, 2008). Metabolomic studies have expanded our understanding of the mechanisms of $C$. reinhardtii adaptation to mineral starvation (Bölling and Fiehn, 2005; Lee et al., 2012), changes in illumination (Mettler et al., 2014), $\mathrm{CO}_{2}$ levels (Renberg et al., 2010; Zhou et al., 2015), and the type of nutrition (Wienkoop et al., 2010; Plancke et al., 2014; Puzanskiy et. al., 2021).

The classical method of revealing trophic plasticity is to place an organism in conditions with a certain set of carbon and energy sources with further physiological, molecular-genetic and biochemical analysis. The main limitation of this method is the lack of complete control over the sources of energy and matter. Such a task can be solved with the help of mutants with defects of the carbon assimilation systems. To identify the significance of the role of $\mathrm{CO}_{2}$ as a carbon source, the CC-981 strain was used in this study, which deficient in phosphoribulokinase ( $p r k 1)$, an enzyme of the Calvin cycle. This mutant needs acetate as carbon source for growth (Moll and Levine, 1970). As was shown earlier, this line lacks PRK protein (Roesler et al., 1992). PRK phosphorylates ribulose-5-phosphate, closing the cycle of regeneration of ribulose-1,5-bisphosphate, which is a substrate of Rubisco (Gurrieri et al., 2021). This strain was grown under illumination in the presence of acetate ( $p r k$-light). Since under such conditions, light and acetate are available energy sources for it, and only acetate can be a carbon source, the status of this culture can be considered as "mixoheterotrophic". It was compared with a heterotrophic culture growing in the dark in the presence of acetate (prk-dark) and a wild-type culture (CC-124) growing under illumination in the presence of acetate (wt-light), which is capable of both photoautrophy and assimilation of acetate as energy and carbon, that is, being mixotrophic.
To determine the value of carbon and energy sources, the dynamics of cell density and biomass density were determined. To reveal the molecular mechanisms of regulation, an RT-PCR expression analysis was performed. To reveal the metabolic features of cultures with different trophic status, in this work, the profiling of metabolites by GC-MS method was carried out.

\section{Material and methods}

\section{Culturing}

Two strains of Chlamydomonas reinhardtii P.A. Dangeard were used. Wild type CC-124 [137c] mt- was obtained from the collection of the Department of Genetics and Biotechnology, Saint-Petersburg State University. The mutant is deficient in phosphoribulokinase CC-981 F60 (prk1) mt- was obtained from Chlamydomonas Resource Center (chlamycollection.org). Batch cultures were maintained in liquid Tris-Acetate-Phosphate (TAP) medium containing $1 \mathrm{~mL} / \mathrm{L}$ glacial acetic acid (Gorman and Levine, 1965) in half-filled $500 \mathrm{~mL}$ bottles on rotary shaker. The CC-124 (wt-light) and CC-981 (prk-light) were cultured under cons-tant illumination with cool-white fluorescent lamps at PAR rate of $80 \mu \mathrm{mol} \mathrm{m}^{-2} \mathrm{~s}^{-1}$. The CC-981 (prk-dark) was also cultured under heterotrophic conditions in the dark. Cell density was measured with hemocytometer.

\section{EXTRACTION}

Sampling and extraction for metabolomics study were performed according to Oliver Fiehn (Bölling and Fiehn, 2005; Lee and Fiehn, 2008) with some modifications (Puzanskiy et al., 2018a). Briefly, a sample of cell culture containing $0.1-0.2 \mathrm{~g}$ of fresh weight was injected into an equal volume of the cold $70 \%$ methanol to quench metabolism rapidly. Then cells were collected by gentle centrifugation ( $3 \mathrm{~min}$ at $5000 \mathrm{~g}, 4^{\circ} \mathrm{C}$ ). The pellet was frozen immediately in liquid nitrogen. Cells were disrupted by bead mill and triple cycle of frozen/thawing in extraction mix. Extraction was made with cold mix of methanol, chloroform and water in ratio of 10:3:1 within 30 min at $4{ }^{\circ} \mathrm{C}$ on termoshaker. Then cell debris was eliminated by centrifugation at $12000 \mathrm{~g}$ for $10 \mathrm{~min}$. Extracts were evaporated in a vacuum concentrator. The dry samples were stored in a freezer at $-80{ }^{\circ} \mathrm{C}$. 
GC-MS ANALYSIS

Firstly, dry samples were dissolved in $100 \mu \mathrm{L}$ of pyridine and $100 \mu \mathrm{L}$ of $99 \%$ BSTFA with $1 \%$ TMCS (Sigma-Aldrich, US) was added for derivatization. Internal standard tricosane (normal hydrocarbon $\mathrm{C}_{23} \mathrm{H}_{48}, 10 \mu \mathrm{L}$ of $1 \mu \mathrm{L} / \mathrm{mL}$ l solution) was added. Then samples were incubated at $90^{\circ} \mathrm{C}$ for $20 \mathrm{~min}$. GC-MS analysis was made using chromatograph Agilent 5860 coupled with Agilent 5975 mass selective detector (Agilent Technologies, US). Separation was performed on a DB-5ms (Agilent Technologies, US) capillary column. The following conditions of chromatography were used: helium flow rate was $1 \mathrm{~mL} / \mathrm{min}$; temperature of evaporator $250{ }^{\circ} \mathrm{C}$ at a splitless mode, initial column temperature was $70^{\circ} \mathrm{C}$, increased by $6^{\circ} \mathrm{C} / \mathrm{min}$ up to $340^{\circ} \mathrm{C}$. Electron impact ionization was performed at $70 \mathrm{~V}$ and an ion source temperature of $230{ }^{\circ} \mathrm{C}$.

\section{GC-MS DATA PROCESSING}

GC-MS data were processed in PARADISe software (Johnsen et al., 2017, Department of Food Science Faculty of Science, University of Copenhagen, Denmark) coupled with NIST MS Search (National Institute of Standards and Technology (NIST), US). In addition, the AMDIS (Automated Mass Spectral Deconvolution and Identification System, NIST, US) was used. Three massspectrometer libraries were used: NIST2010 (NIST, USA), the Golm Metabolome Database (Max Planck Institute for Molecular Plant Physiology), and in-house library of the Laboratory of Analytical Photochemistry (Botanical Institute RAS). Retention index (RI) was determined by calibration with standard alkanes.

\section{RNA ISOLATION}

$10^{7}-10^{8}$ cells were collected by centrifugation (5 min, $3000 \mathrm{~g}, 4^{\circ} \mathrm{C}$ ), and resuspended in $1 \mathrm{~mL}$ of PureZOL ${ }^{\mathrm{TM}}$ (Bio-Rad, US) and mixed. Homogenate was frozen and thawing, and incubated at room temperature for 5-10 $\mathrm{min}$. Then, cell debris was eliminated by centrifugation at $12000 \mathrm{~g}$ for $10 \mathrm{~min}$, at $4{ }^{\circ} \mathrm{C}$. After that, $0.2 \mathrm{~mL}$ of chloroform was added and the mix was agitated for $15-20 \mathrm{~s}$ and incubated for $5 \mathrm{~min}$ at room temperature with periodic stirring. Phases were separated by centrifugation at $4{ }^{\circ} \mathrm{C}$ during $15 \mathrm{~min}$ at $12500 \mathrm{~g}$ and aqueous phase was collected. Then $0.5 \mathrm{~mL}$ of isopropanol was added, and samples were incubated for $10 \mathrm{~min}$ at room temperature. The sample was then centrifuged for 10 $\min$ at $4{ }^{\circ} \mathrm{C}$ at $12500 \mathrm{~g}$ and supernatant was removed. The pellet was washed by adding $1 \mathrm{~mL}$ of $75 \%$ ethanol, vortexed and centrifuged for $5 \mathrm{~min}$ at $4{ }^{\circ} \mathrm{C}$ and $12500 \mathrm{~g}$ and the supernatant was discarded. The pellet was dried a little and dissolved in DEPC water. Quality and quantity of isolated RNA was checked spectrophotometrically by the absorption ratios in wavelengths of 260/230 and 260/280 nm using a NanoDrop 1000 spectrophotometer (Thermo Fisher Scientific, US). The samples were stored at $-80^{\circ} \mathrm{C}$.

\section{CDNA SYNTHESIS}

RNA was purified from DNA using DNase I, RNase-free (Thermo Fisher Scientific, US) according to the vendors's manual. cDNA was synthesized using Oligo(dT) primers (BioBeagle, Russia) and $\mathrm{M}-\mathrm{MuLV}$ reverse transcriptase (Thermo Fisher Scientific, US) according to the vendor's instructions with the application of RiboLock RNase inhibitor (Thermo Scientific, US). The samples were stored at $-80{ }^{\circ} \mathrm{C}$.

\section{Real time PCR}

Primers were designed using the Beacon-Designer 8 program (Sigma Aldrich, US). The length of primers was approximately 20 nucleotides, the length of amplicon was 80-250 nucleotides, predicted melting temperature was approximately $60{ }^{\circ} \mathrm{C}$. Sequences of primers used in this work are shown in Table 1 . The quality of primers was estimated by the analysis of PCR products using PAG electrophoresis as well as the analysis of curves of melting and the accumulation of products during real time PCR. RT-PCR was performed with SYBR Green I supermix (Sintol, Russia) using a CFX96 real-time PCR System (BioRad, US) under the following conditions: $7 \mathrm{~min}$ at $95^{\circ} \mathrm{C}$, then 40 cycles at $95^{\circ} \mathrm{C}$ for $15 \mathrm{~s}$, and at $60{ }^{\circ} \mathrm{C}$ for $50 \mathrm{~s}$.

\section{ReAL TIME PCR QUANTIFICATION}

Two genes were used as references. The first gene $C B L P / R A C K 1$ encoding $\mathrm{G}$ protein $\beta$ subunit-like polypeptide is the most common reference gene in $C$. reinhardtii transcription investigations (for example, see: Fang et al., 2012; Ostroukhova and Ermilova, 2019). The second one is RPL19encoding ribosomal protein L19, which stable expression 
Table 1. Brief description of the genes and sequences of primers, which expression has been analyzed.

\begin{tabular}{|l|l|l|l|l|}
\hline \multicolumn{1}{|c|}{ Name } & \multicolumn{1}{|c|}{ Locus tag } & \multicolumn{1}{c|}{ Primers } & \multicolumn{1}{c|}{ Product } \\
\hline RBCS1 & CHLREDRAFT_82986 & $\begin{array}{l}\text { CTGAAGCCGCCGTCAAGGAG } \\
\text { AGGAGGCAGGTAGGAGAAGGT }\end{array}$ & Rubisco small subunit 1 & carbon fixation \\
\hline CIS1 & CHLREDRAFT_194915 & $\begin{array}{l}\text { TACATCCCGCCCTCCTACAACCT } \\
\text { ACCCGCCAGCCCGTTCAT }\end{array}$ & citrate synthase 1 & TCA beginning \\
\hline CIS2 & CHLREDRAFT_194915 & $\begin{array}{l}\text { AACACCACCGCCGTCATCTCAC } \\
\text { ATCAATCACCTCCGTCG }\end{array}$ & citrate synthase 2 & glyoxylate cycle beginning \\
\hline APE2 & CHLREDRAFT_205633 & $\begin{array}{l}\text { GCTCGTCCGTGCTGTTCTTCAC } \\
\text { CTTCTTGTGCTTGTGCTTGT }\end{array}$ & triose phosphate translocator & triose phosphate shuttle \\
\hline OMT1 & CHLREDRAFT_185488 & $\begin{array}{l}\text { GGAGTGCCTGAACAACAACAG } \\
\text { AAGTAGTGCGATAGAAGT }\end{array}$ & $\begin{array}{l}\text { oxoglutarate (oxaloacetate): } \\
\text { malate antiporter }\end{array}$ & $\begin{array}{l}\text { malate valve, plastid nitrogene } \\
\text { metabolism }\end{array}$ \\
\hline AAA1 & CHLREDRAFT_195230 & $\begin{array}{l}\text { GCCGTGCCTGCTGTTGCCGCTC } \\
\text { GCCCAGACCACCCTT }\end{array}$ & plastidic ADP/ATP translocase & direct ATP transport into plastid \\
\hline CBLP/RACK1 & CHLREDRAFT_105734 & $\begin{array}{l}\text { GTCATCCACTGCCTGTGCTTCC } \\
\text { TCTTGCTGGTATGTTG }\end{array}$ & $\begin{array}{l}\text { receptor of activated protein } \\
\text { kinase C }\end{array}$ & reference gene \\
\hline RPL19 & CHLREDRAFT_195590 & $\begin{array}{l}\text { TCGGTCTGAACTGCGGTCTGC } \\
\text { GGGCTGCTACGGAAGATGA }\end{array}$ & ribosomal protein L19 & reference gene \\
\hline
\end{tabular}

was shown earlier (Liu et al., 2012). Expression was determined using the $2^{-\Delta \mathrm{Ct}}$ method (Livak and Schmittgen, 2001), and the final relative quantity was calculated as geometric mean of values for two reference genes. The measurements were carried out in three biological replicates.

\section{DATA ANALYSIS AND VISUALIZATION}

For statistical analysis, the R-language environment (http://www.R-project.org/) was applied. Metabolomics data were normalized per sample median, log-transformed and standardized. Outlying values were excluded based on Dixon's test. If a value was excluded or was zero but it was not zero in other replications, it was postulated as randomly missed and was imputed with KNN (k-nearest neighbors) method from the impute R-package (Hastie et al., 2019). The remaining zeroes were substituted by $1 / 10$ of the minimum values. For Principal Component Analysis (PCA) we used R-package pcaMethods (Stacklies et al., 2007). Partial Least Square Discriminant Analysis (PLSDA) was performed in ropls package. VIPs (Variable Importance in Projection) values were used for feature selection (Pérez-Enciso and Tenenhaus, 2003; Therien et al., 2014). Random Forest (RF) was hold by random Forest (Liaw and Wiener, 2002). The heatmap of the metabolites was made with ComplexHeatmap package (Gu et al., 2016). Graphs were built and analyzed with Cytoscape software environment (Shannon, 2003).

\section{Results}

\section{Cultures' Growth}

The growth of cell density (Fig. 1, A) and fresh biomass (Fig. 1, B) during the log phase was approximately equal for prk-light and wt-light, which indicates a non-critical value of carbon fixation for growth. At the same time, the growth of $w t$-light reached its maximum values already at the first day, while the growth of prk-light lagged behind. Moreover, if the growth of cell density in the first day was practically absent, then the growth of biomass took place, although it was noticeably lower than that of $w t$-light. In final cell density $w t$-light slightly exceeded prk-light $(P=0.004)$. It should also be noted that the weak growth of raw biomass continued after the end of proliferation. Thus, wt-light cells accumulated a large biomass $(P=0.002)$. In turn, dark growing $p r k$-dark cultures demonstrated several times lower growth rate and final biomass, as well as cell density, compared to $w t$-light cells (Fig. 1).

\section{Metabolomic ANALYSIS}

\section{General characteristics}

The metabolic profiles of $C$. reinhardtii detected by the GC-MS method included 150 metabolites, of which 59 compounds were identified and a chemical class was determined for another 13. Amino 

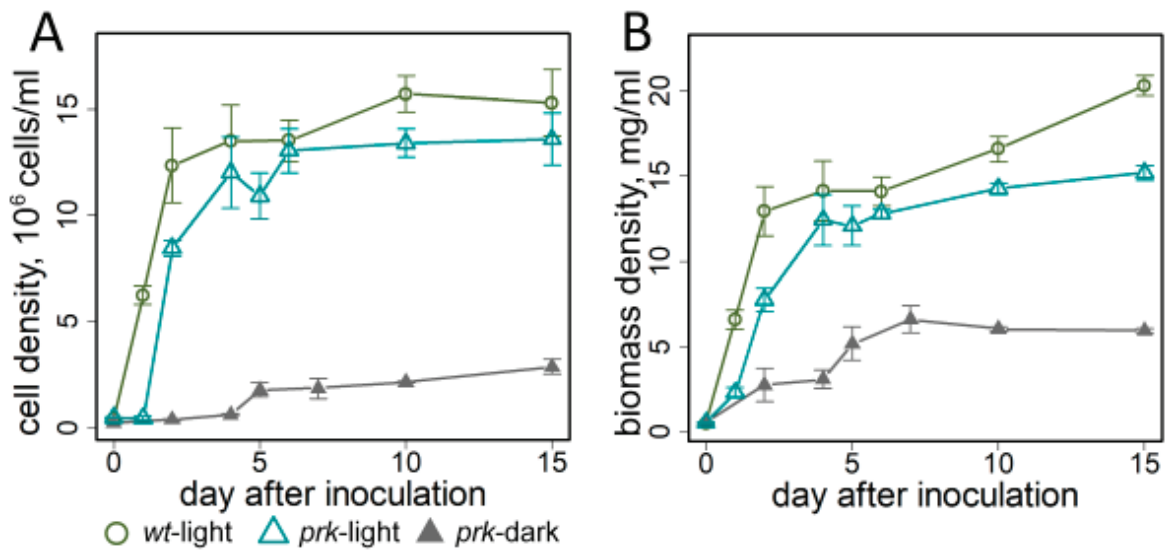

Fig. 1. Growth of Chlamydomonas reinhardtii batch cultures: (A) cell density, $10^{6}$ cells per $\mathrm{mL}$, (B) biomass density expressed as mg of fresh weight per $\mathrm{mL}$. Bars are $95 \%$ confidence intervals; wt-light - mixotrophic culture of wild type CC-124 supplied with light and acetate; prk-light - mixoheterotrophic culture of phosphoribulokinase deficient mutant CC-981 supplied with light and acetate; prk-dark - heterotrophic culture of CC-981 supplied with acetate in the dark.

acids (19), including 14 proteinogenic ones, were widely represented in the obtained profiles. Other nitrogen-containing compounds (7) were mainly represented by various amines and amides that are intermediates of nitrogen exchange. About a dozen carboxylic acids have also been identified, including intermediates of energy metabolism. Lipophilic compounds were represented by a dozen fatty acids from $\mathrm{C} 9$ to $\mathrm{C} 18$ and their acylglycerols. Several terpene-like and ergosterol-like compounds have also been identified. Most of the 17 identified carbohydrates were represented by complex sugars, including sucrose. Several sugar alcohols and a small amount of hexoses were also noted.

At the first stage, in order to identify the similarity of the metabolic profiles, they were visualized in a low-dimensional space, using unsupervised methods. For this purpose, the PCA (Principal Component Analysis) method was used. Figure 2, A shows the scattering of samples in the score space of the first two PCs, explaining 38.1 and $14.8 \%$ of the variance, respectively. Using the content of metabolites as a measure of proximity is not always the best solution, since it may depend on the method of normalization. Therefore, as a measure of the proximity of the metabolic profiles, the correlation in the content of metabolites was considered, the dimension was reduced using MDS (Multidimensional scaling). It can be seen that in both the first and second models, the metabolic profiles are clearly divided into 3 groups corresponding to the trophic status.

\section{Classification}

At the next stage, the classification and evaluation of the correlation of the metabolites levels with the difference between the classes was carried out by the PLS-DA (Partial Leasts Squares - Discriminant Analysis) and RF (Random Forest) methods. The resulting PLS-DA model was characterized by the following parameters: $\mathrm{R}^{2} \mathrm{Y}=0.966(P \leq 0.005)$, $\mathrm{Q}^{2} \mathrm{Y}=0.944(P \leq 0.005)$ that indicate its high statistical reliability. The model included two predictive components that were associated with $38 \%$ and $15 \%$ of the variance, respectively. The relation of metabolites to class differences in the PLS-DA classification was evaluated by the VIP value (Variable Importance in Projection). For RF, $100 \%$ classification accuracy was achieved. The assessment of the association of metabolites with the differences in cultures during classification by the RF method was determined by the MDA value (Mean Decrease Accuracy).

As shown in Figure 3, the dissimilarities in cul-tures with different trophic status concerned a wide range of compounds, including amino acids, sugars, lipids and carboxylates. The main characteristic feature of prk-light cultures was the relatively high content of fatty acids, especially C16-C18 and lipophilic compounds associated with the metabolism of terpenes, including ergosterol. In addition, it should be noted that the prk-light culture was distinguished by a slightly higher level 

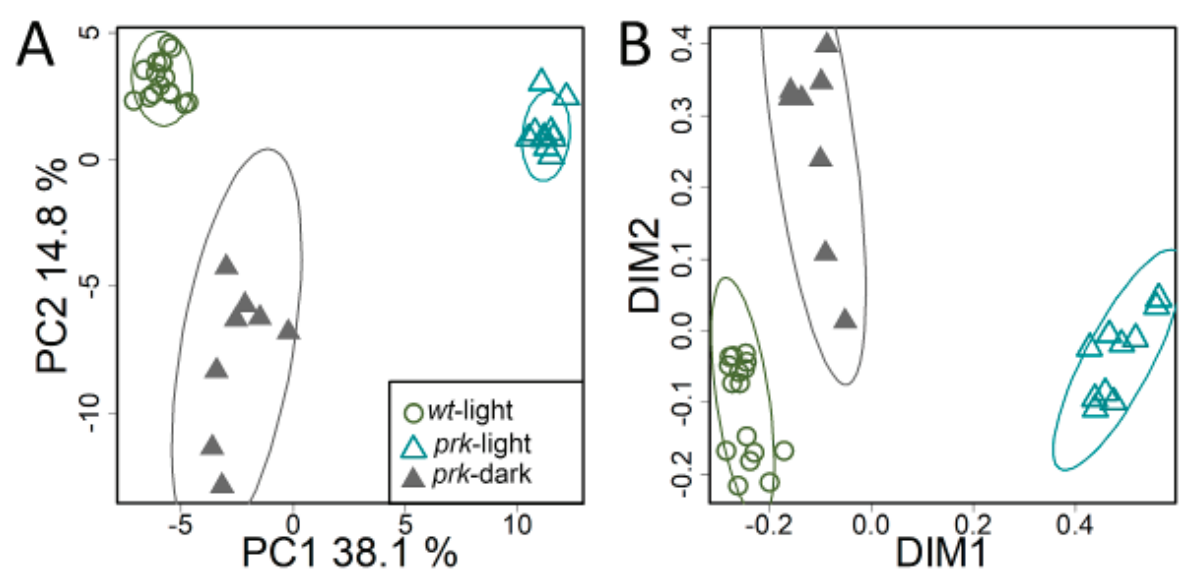

Fig. 2. Representation of metabolic profiles in lower dimensional spaces: (A) PCA score plot, $\%$ - the variance associated with the PC, (B) representation of metabolite profiles in the space obtained using MDS (multidemensional scaling) with Pearson distance (1-r). Ellipses are 95\% confidence intervals.

of a number of sugar alcohols and complex sugars. It was possible to register an increased level of glycolate, malate and glutamate. The prk-dark cells were characterized by a relatively elevated level of compounds involved in nitrogen exchange, such as urea cycle intermediates: arginine, ornithine and urea, as well as the product of arginine metabolism - guanidine. The prk-dark culture was also characterized by a high level of such a key component in energy metabolism as citrate. Some complex sugars, including sucrose, showed higher levels in prk-dark. The accumulation of metabolites was less typical for $w t$-light. For $w t$-light, a higher level of amino acids was established, primarily threonine, asparagine and valine.

\section{Metabolite networks}

To clarify the connection of trophic status with systemic metabolic networks, a correlation analysis of the metabolite levels was carried out. The result is presented in the form of graphs (Fig. 4), where the nodes correspond to the metabolites, and the edges correspond to the significant Spearman correlation $(P<0.01)$. The edges corresponding to the positive correlation "pull together" the metabolites, and the negative ones - "push apart". All three networks showed a scale-free structure. The similarity of the obtained networks is the presence of regions of lipophilic compounds and regions containing mainly carboxylates and amino acids. However, the structure of the networks differentiated significantly. This indicates significant distinctness in metabolic connections in cells of different trophic status. An important criterion for the dissimilarity between networks was their diameter; it was similar for $w t$ light (8) and prk-light (10) and was significantly larger for prk-dark (14). In addition, the network heterogeneity of prk-dark was higher $(0.814)$ than that of $w t$-light (0.635) and prk-light (0.663). The length of the characteristic path also differed and was minimal for wt-light (2.9) and maximal for prkdark (4.3), prk-light showed an average result (3.5).

\section{GeNe EXPRESSION}

The relative expression level of the $R B C S 1$ gene encoding the small Rubisco subunit was higher in all the analyzed variants during the period of culture density growth (Fig. 5). At the same time, the expression level in growing prk-light cultures exceeded that of the other two by several times. Nevertheless, at the end of the growth, the expression level fell and the differences were leveled.

Both genes CIS1, 2 encoding citrate synthases had the maximum expression level in the actively growing $w t$-light cultures. The expression level of CIS1 encoding citrate synthase associated with TCA was higher during the growth period in prklight cultures, but it was several times lower than that of $w t$-light cultures. Although the expression of CIS1 in wt-light decreased after the end of growth, it exceeded that in other cultures. At the same time, the level of CIS2 expression in wt-light decreased approximately to the level of other cultures, in which it remained relatively low all the time. It should be noted that a slightly different pattern of CIS1,2 expression dynamics was observed in prk- 


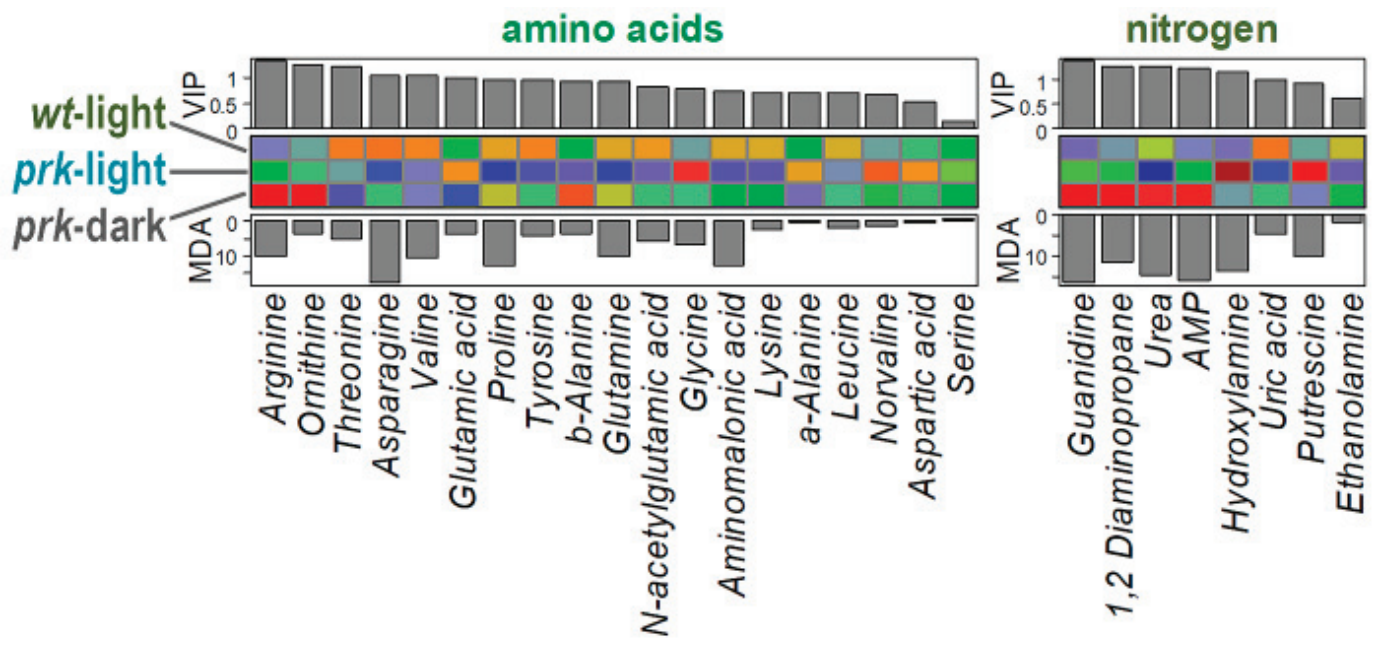

carboxylates and others

sugars
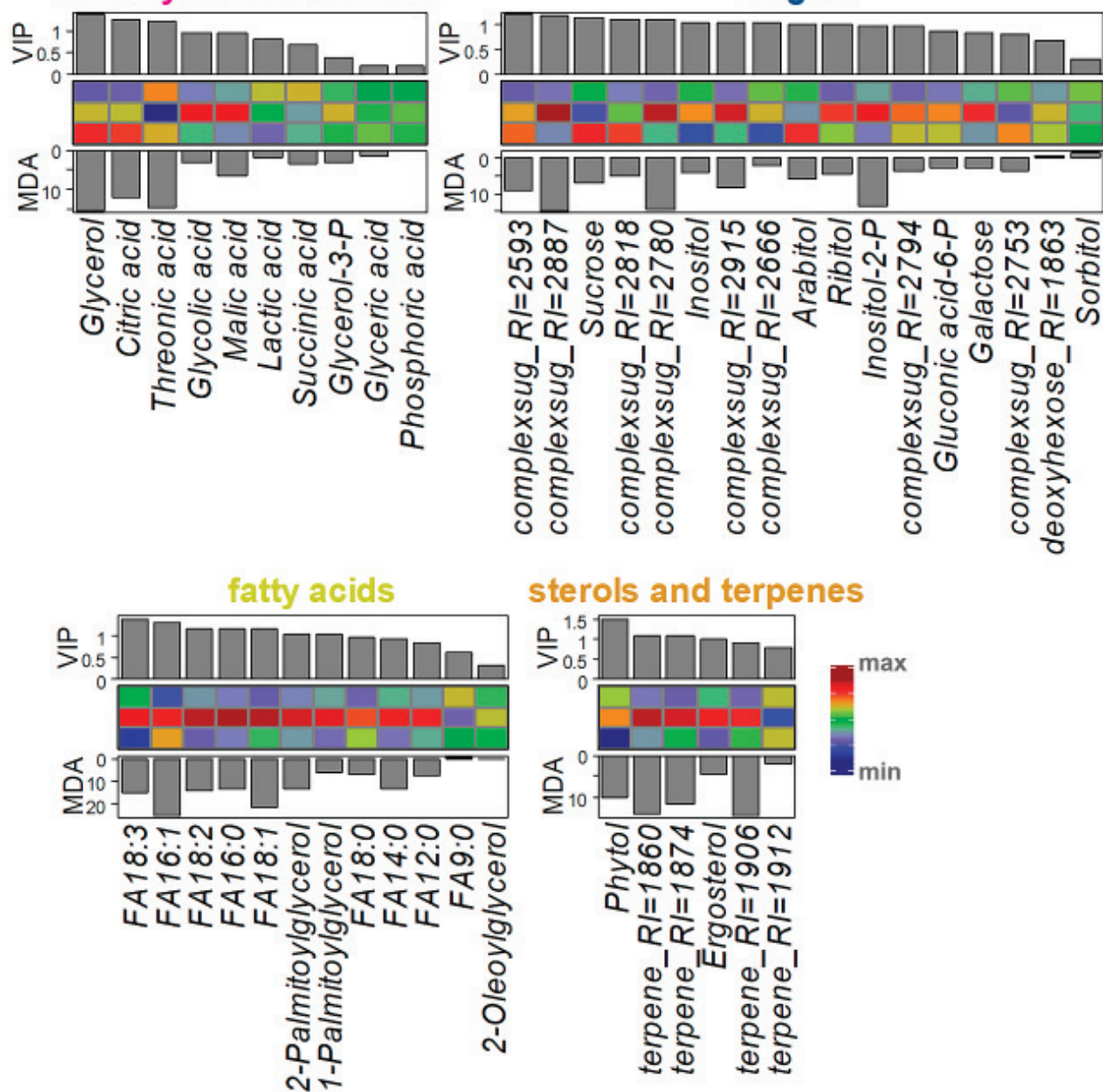

sterols and terpenes

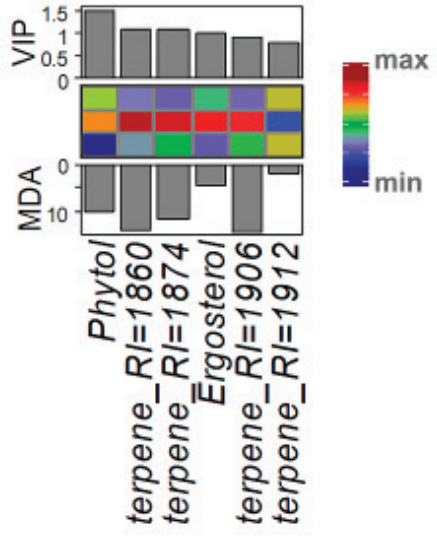

Fig. 3. Heatmap of mean arbitrary content of identified metabolites in Chlamydomonas reinhardtii batch cultures. Culture names as in Fig. 1. Data are normalized per sample median, log-transformed and standardized. In metabolite names: "RI" - retention index, "P" - phosphate, "complexsug" - oligosaccharides or other molecules with sugar parts. Bars above - VIPs from PLS-DA model, bars at the bottom - mean decrease accuracy (MDA) from Random Forest. 


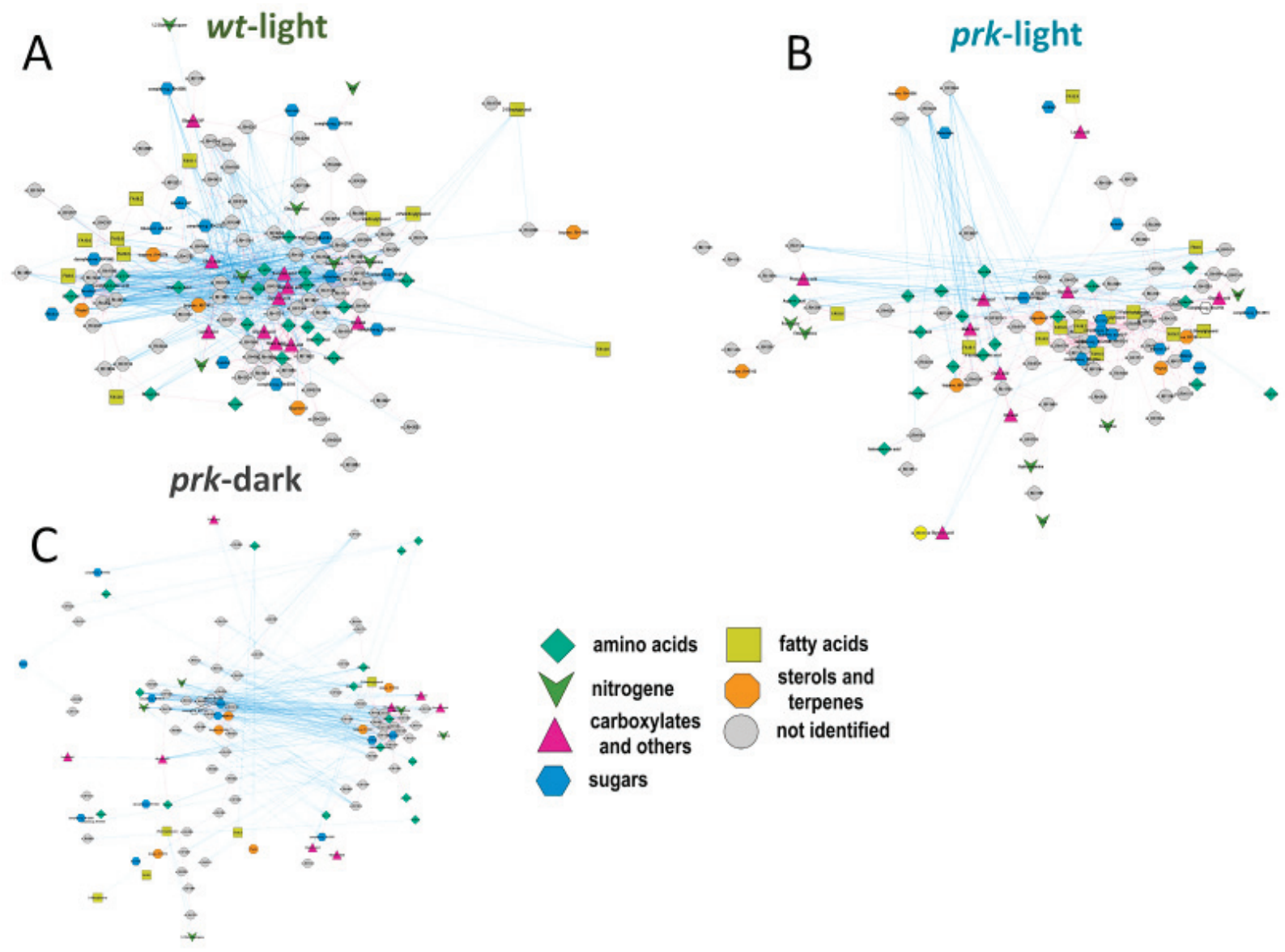

Fig. 4. Mapping of metabolites by correlations. The nodes are metabolites; edges are significant Spearman's correlations $(\mathrm{P}<0.01)$. The shape and color reflect the chemical nature of the compound; names are the same as in Fig.1.

dark. The expression level of these genes was low at first, and then increased slightly after the growth was completed.

The maximum expression level of the $A A A 1$ gene encoding the ATP/ADP antiporter was detected in prk-dark at the growth phase, followed by a decrease in its level. A similar pattern was observed in $w t$-light, but the expression level was lower. The minimum expression level was shown by prk-light cells.

In the case of genes encoding the transporters of the malate valve $O M T 1$ and the triosophosphate shunt APE2, the maximum expression level was shown by $w t$-light at the beginning of the exponential growth phase with its subsequent decrease. In the case of APE2, also in the growth phase, the level was higher, and it was equal to that of $w t$-light in the middle of the growth phase, and then fell. The minimum level of expression in the growth phase was shown by prk-light cells. In the case of OMT1, prk-light cells had a weak induction of expression at the beginning of the growth phase, but it was still many times lower than in $w t$-light.

\section{Discussion}

\section{GROWTH}

The question of the contribution of the autoand heterotrophic component to energy and carbon budgets is a key one in the study of trophic adaptation of mixotrophic microalgae. Despite the fact that $C$. reinhardtii in the presence of a substrate retains a functional photosynthetic apparatus, its value for growth may vary depending on trophic conditions. The addition of acetate during the enrichment of the $\mathrm{CO}_{2}$ medium and high illumination intensity does not lead to an increase in the growth rate (Heifetz et al., 2000). It can be concluded that the photosynthetic apparatus provides cells with energy 

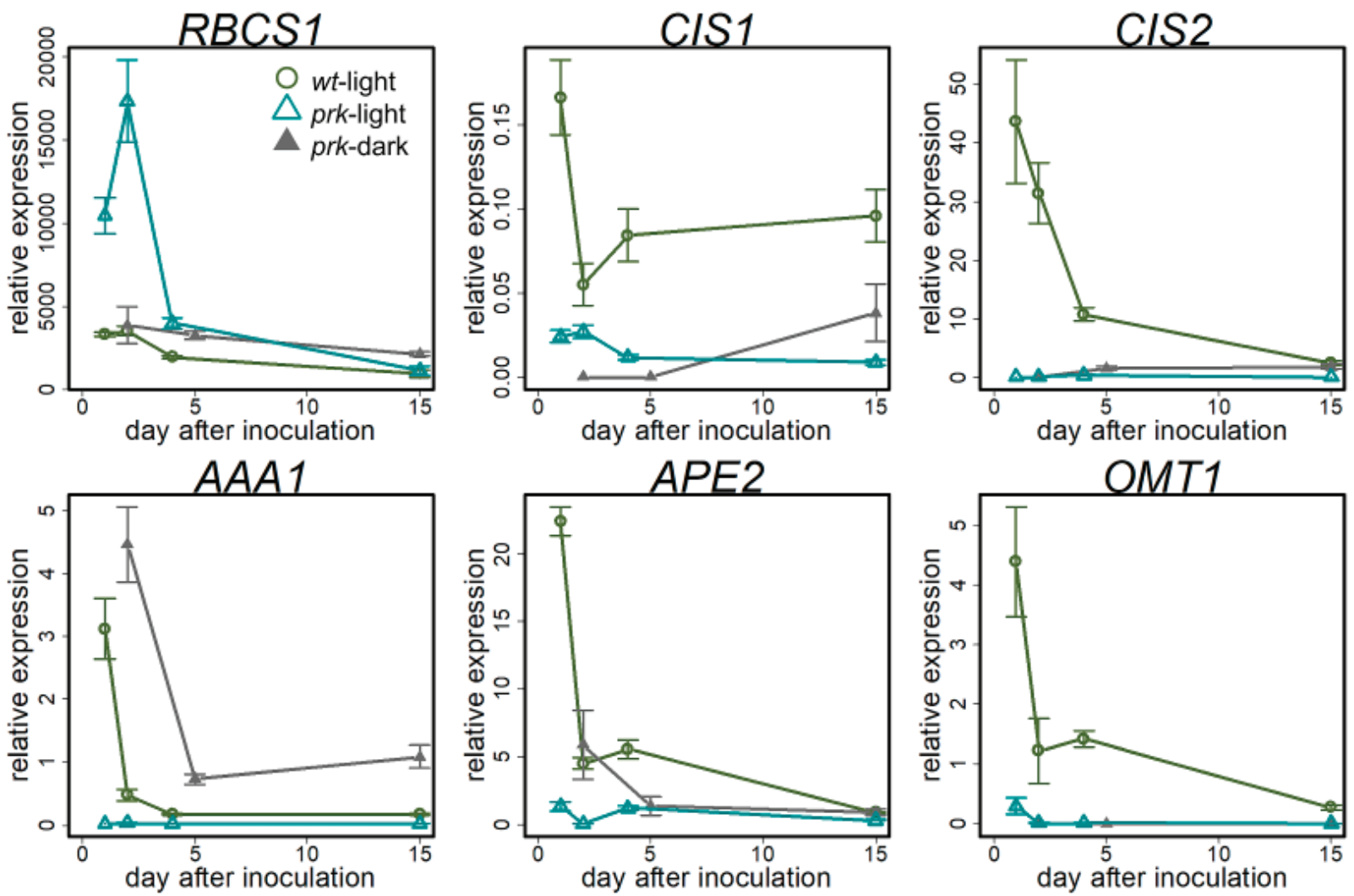

Fig. 5. Trophic status effects on the dynamics of relative expression of six genes encoding enzymes and plastid transporters involved in carbon metabolism during batch cultures of $C$. reinhardtiigrowth. Bars are $95 \%$ confidence intervals. Cultures are marked as in Fig. 1. Short description of the genes is given in the Table 1.

and carbon to an extent sufficient for maximum growth. Under conditions that limit photosynthetic activity, the growth rate and the final biomass increase dramatically when an exogenous substrate is added. Thus, the possibilities of photosynthesis may not always fully meet the needs of cells (Fischer et al., 2006; Puzanskiy et al., 2021). Under optimal conditions for photosynthesis, the presence of acetate in the medium leads to a decrease in the level of $\mathrm{CO}_{2}$ fixation compared to autotrophy (Hudock and Levine, 1964; Togasaki and Levine, 1970; Heifetz et al., 2000), which indicates a change in carbon uptake. The conclusion that acetate is an important factor in the growth of biomass during mixotrophy is also supported by the fact that a mutation leading to the loss of function by the enzyme isocitrate lyase, necessary for the assimilation of carbon from acetate, causes a decrease in the growth rate by several times (Plancke et al., 2014).

Figure 1 shows the results of comparing the dynamics of cell density and biomass of $w t$-light - mixotrophic and prk-light - mixoheterotrophic cultures. In the case of a mutant, light and acetate are used as energy sources, but acetate is the sole source of carbon, since the cells are not capable of fixing $\mathrm{CO}_{2}$. It can be seen that the loss of the ability to fix $\mathrm{CO}_{2}$ practically does not affect the growth rate in the exponential phase, which indicates that the contribution of photosynthesis to the carbon budget in mixotrophic cultures of $C$. reinhardtii can be compensated by an exogenous substrate. This may be due to the low contribution of $\mathrm{CO}_{2}$ fixation to the growth of biomass during mixotrophy. This is consistent with the data showing that mutations leading to the loss of carbon-concentrating mechanisms do not affect the growth rate of mixotrophic $C$. reinhardtii cultures (Yun et al., 2021). However, the transfer of prk-dark culture to darkness leads to a sharp decrease in the rate of culture density accumulation and final biomass. This finding is consistent with the previously known data on the low growth rate of heterotrophic $C$. reinhardtii cultures (Sager and Granick, 1953).

It can be concluded that for $C$. reinhardtii cultures, acetate is the main source of carbon, and photosynthesis is the most important source of energy. 
The revealed ability of $w t$-light cells to accumulate biomass after stopping proliferation was observed earlier in cultures of various microalgae, for example, in Botryococcus braunii (Belcher, 1968) and Haematococcus pluvialis (Zhekisheva et al., 2002). The accumulation of biomass in stationary cultures is primarily associated with the accumulation of reserve compounds. It has been shown that in autotrophic C. reinhardtii cultures, after the end of growth, the phase of lipid accumulation is revealed (Lv et al., 2013). It is known that the transition of C. reinhardtii culture to the stationary phase is caused by the exhaustion of acetate (Singh et al., 2014; Therien et al., 2014; Bogaert et al., 2019); therefore, the accumulation of lipids after the completion of growth is provided by photosynthetic activity. This fact may explain the lack of biomass growth in stationary prk-light cultures, since they are not able to fix $\mathrm{CO}_{2}$. The higher level of accumulated storage compounds may explain the faster start of growth after inoculation in $w t$-light compared to prk-light. The low rate of autotrophic biomass growth in aging cultures may be also due to a decrease in the level of photosynthetic activity (Samuelsson and Oquist, 1977; Puzanskiy et al., 2018a).

\section{Rearrangement of the Krebs cycle (TCA)}

Since the loss of the ability to fix $\mathrm{CO}_{2}$ makes acetate the only source of carbon, it can be expected that cells will develop metabolic adaptations to optimize their metabolism. The assimilation of acetate begins with the formation of acetyl-CoA, which can be directed to the synthesis of fatty acids or to the TCA, where it can be oxidized or incorporated into metabolism through a glyoxylate shunt (Johnson and Alric, 2013). It has been shown that the presence of acetate in the medium induces the expression and activity of glyoxylate cycle enzymes (Harrop and Kornberg, 1966; MartínezRivas and Vega, 1993; Hayashi et al., 2015). The glyoxylate pathway is a modification of the TCA, where the decarboxylating part is cut off by the conversion of isocitrate to malate. The latter is then converted to oxaloacetate, which can leave the cycle and be directed to gluconeogenesis (Boyle et al., 2017). The conversion of malate to oxaloacetate is a reversible reaction, from this point its direction is determined by the content of substrates. It is known that pools of carboxylates, including malate, play an important role in the regulation of activity of TCA (Igamberdiev and Eprintsev, 2016; Igamberdiev and Bykova, 2018). A high level of malate in prk-light should lead to a shift in the equilibrium towards oxaloacetate and promote metabolic flow towards gluconeogenesis. The source of malate in this case is probably the activity of the glyoxylate shunt (Nogales et al., 2004). Alternatively, oxaloacetate can be converted to citrate by the enzyme citrate synthase because of another reversible reaction. Similarly to malate, citrate pool also plays an important role in regulation of TCA activity and operating mode (Igamberdiev and Eprintsev, 2016; Igamberdiev and Bykova, 2018). Therefore, as in the case of malate, a high level of citrate should shift the balance towards oxaloacetate, which will also contribute to the flow of carbon into gluconeogenesis. At the same time, the reduced expression of the genes encoding citrate synthases in prk-light may also contribute to the release of oxaloacetate into gluconeogenesis.

Thus, it can be concluded that the biochemical and molecular features of prk-light provide, first of all, the outflow of carbon acetate from the glyoxylate cycle (and the TCA as a whole). The price of this event is a possible decrease in the level of entry into the glyoxylate cycle/TCA. This process most likely compensates for a decrease in the activity of the oxidative part of the TCA due to a decrease in its role in the energy balance. This happens due to an increase in the photosynthesis part in it, since carbon fixation in prk-light does not work and does not consume energy. Besides, maintaining a high flow through the TCA with a decrease in the activity of citrate synthases can be ensured by a decrease in the activity of citrate lyases, which is catalyzed by the decomposition of citrate into oxaloacetate and acetyl-CoA (Plancke et al., 2014). It was previously shown that with autotrophy, when there are few acetyl groups, there is a decrease in the expression level of genes encoding isocitrate lyases (Puzanskiy et al., 2020a). Probably, a similar situation develops with an increase in the consumption of acetyl groups in gluconeogenesis.

With prk-dark heterotrophy, higher citrate content was observed, but the level of malate was significantly lower. On the one hand, the greater accumulation of citrate is probably due to an increase in the flow to the TCA, since it now serves not only as a site for redirecting carbon to gluconeogenesis, but also as the main energy supplier. On other hand, high level of citrate could be explained from fact that TCA is a main source of reducing power in the dark, which could be exported from mitochondria through citrate valve. This process consists of export citrate and its oxidation in other compartments (Igamberdiev and Eprintsev, 2016; Igamberdiev and 
Bykova, 2018). The decrease in the level of malate in heterotrophy is probably associated with a low flow through the glyoxylate shunt under heterotrophy. For many algae, it has been shown that a much larger proportion of the substrate is oxidized in the dark than when illuminated (Goulding and Merrett, 1966, 1967). Modeling of the heterotrophic metabolism of C. reinhardtii showed that only $20 \%$ of the carbon entering the TCA is sent to the glyoxylate shunt and the major part is oxidized (Boyle et al., 2017).

It should be noted that the maximum expression levels of genes of citrate synthases and other analyzed genes were involved in central metabolism during the period of log-phase, which is characterized by the maximum metabolic activity. A similar picture has already been observed earlier under both mixotrophic (Puzanskiy et al., 2020b) and autotrophic conditions (Puzanskiy et al., 2018b).

\section{ENERGY TRANSPORT}

Blocking of carbon fixation reduces the energy requirements of the plastid, which is the reason for a sharp decrease in the expression of the $A A A 1$ gene encoding the transporter that supplies ATP to the plastid. Interestingly, the level of $A A A l$ in the dark, on the contrary, increased and was maximum, which is obviously due to the need to supply the plastid with energy. In the case of heterotrophy, the balance of ATP in chloroplasts is negative or zero, even with active glycolysis and OPPP; therefore, in the dark, ATP should be imported into the chloroplast (Johnson and Alric, 2013). It is known that when ethanol is added to the $C$. reinhardtii culture, the level of $A A A 1$ increases. The induction of this protein synthesis is probably part of the mechanism compensating for the repression of ATP synthesis in the chloroplast caused by ethanol, which is toxic to $C$. reinhardtii (Jiang et al., 2017).

Alternatively, the level of APE2 expression also decreased in $p r k$-light, which indicates a lowering in the activity of the triose phosphate shuttle, which provides the exchange of reducing power between the plastid and cytosol. The reason for this may be dysfunction of the Calvin cycle and increased flow through glycolysis/gluconeognesis towards synthesis. At the same time, one of the stages of gluconeogenesis and the Calvin cycle are involved in the triose phosphate shuttle: the reversible conversion of 3-PGA to G3P. Thus, maximizing the flow through this reaction, despite the fact that the Calvin cycle is no longer a source of 3-PGA, can be associated with disabling the triose phosphate shuttle at prk-light. Another situation was observed in the dark: the expression of $A P E 2$ was significantly higher than that of prk-light in the growth phase, which is probably due to the critical need to supply the plastid with reduced cofactors, even at the cost of diverting resources from gluconeogenesis.

A shift in the balance of the conversion of malate and oxaloacetate towards the latter and its supposed outflow into gluconeogenesis should lead to dysfunction of the malate valve, since its activity is based on the reversibility of reactions. It is possible that this happens because of a decrease in the expression level of the gene encoding the plastid antiporter malate OMT1 in prk-light and prk-dark. Interestingly, the suppression of $O M T 1$ expression in tobacco led to the accumulation of carboxylates, including citrate and malate (Schneidereit et al., 2006), as in the case of prk-light.

\section{Nitrogen EXCHANGe}

Changes in the activity of the TCA, the exchange and transport of dicarboxylates also affect the level of nitrogen metabolism and amino acid synthesis. Thus, malate and the OMT1 transporter play an important role in the integration of plastid and cytosolic nitrogen metabolism (Weber and Flügge, 2002; Eisenhut et al., 2015). Engaging of malate for providing an intensive flow to oxaloacetate and a decrease in the downregulation of $O M T 1$ can lead to a decrease in the rate of glutamate withdrawal from the plastid in exchange for carboxylates. This should have a negative impact on nitrogen metabolism. Besides, the consequence of an increased level of carbon flow through the glyoxylate cycle into gluconeogenesis should be a decrease in the direction of carbon skeletons in the synthesis of amino acids. All this probably leads to a decrease in the level of amino acids in prk-light. Previously, a decrease in the level of amino acids has already been shown when OMT1 expression was suppressed in higher plants (Schneidereit et al., 2006; Kinoshita et al., 2011).

In the dark, on the contrary, there is an accumulation of nitrogen exchange intermediates. It was shown earlier that the main product of assimilation of acetate by Chlamydomonas mundana acetate in the dark were amino acids (Eppley et al., 1963). The reason for the increase in the level of amino acids may be a decrease in the metabolic flow through the glyoxylate shunt and increased generation of carbon skeletons in the Krebs cycle. A similar pattern is 
observed in the $C$. reinhardtii mutant, which has lost isocitrate lyase and, as a result, the functional activity of the glyoxylate shunt (Plancke et al., 2014). High level of nitrogen metabolism may be supported by export of citrate, which level is maximal in the dark growing cells, from mitochondria (Igamberdieva and Bykova, 2018). Accumulation of amino acids and nitrogen compounds may arise from low level of biomass accumulation because of low intensity of protein synthesis. The low level of culture density growth may be associated with the need to utilize most of the acetate for energy generation (Boyle et al., 2017). In addition, the energy flow in heterotrophic cells is small in the absence of photosynthesis, which is indicated by a high level of AMP.

\section{Photorespiration, glycolate and Rubisco}

An unusual effect of Calvin cycle dysfunction was a dramatic increase in the expression level of the $R B C S 1$ gene encoding the small Rubisco subunit. This effect may be due to the fact that the expression of Rubisco is controlled by negative feedback from photosynthetic products (Krapp et al., 1993; Ono and Watanabe, 1997), the accumulation of which is blocked. At the same time, the activity of Rubisco is controlled by the oxidative status, which depends on the ETC of plastids and, consequently, lighting (Oswald et al., 2001); therefore, an increase in the expression level of $R B C S 1$ was not observed in the dark.

A distinctive feature of prk-light is the high level of glycolate. This may be a sign of photorespiration activation. This pattern is observed in the case of a disturbance of the Rubisco carboxylating function, for example, as a result of a decrease in the level of $\mathrm{CO}_{2}$ (Renberg et al., 2010) or a defect in the carbon-concentrating mechanisms (Yun et al., 2021). Glycolate can be converted into malate. This is in accordance with lower accumulation of malate in prk-dark growing in the dark and not showing increased expression of $R B C S 1$. The hypothetical high activity of photorespiration is consistent with an increase in the level of $R B C S 1$ expression. This assumption is called into doubt by the fact that the prk mutation, which prevents the regeneration of RBF, blocks both the carboxylating and oxidative functions of Rubisco. We can only assume that there is a weak alternative, possibly non-specific source of RBF. This is indirectly indicated by the very low phosphoribulokinase activity in this mutant (Moll and Levine, 1970), while the enzyme itself was not detected (Roesler et al., 1992).

\section{ACCUMULATION OF FATTY ACIDS}

The effect accompanying the reduced expression of CISI, 2 is the fatty acids accumulation in prklight. This is consistent with the data showing that the activity of citrate synthases plays a key role in the distribution of acetyl groups between the synthesis of triacylglycerols (TAG) and TCA. Inhibition of the activity of this enzyme through antisense repression of genes led to an increase in the level of TAG by more than one and a half times. Overexpression led to a decrease of TAG level by two times (Deng et al., 2013). It can be assumed that the accumulation of fatty acids in prk-dark cells was not observed because the oxidation of acetyl groups for energy supply prevented the accumulation of reserve compounds.

\section{CORRELATION NETWORKS}

An important aspect of system-biological research is the analysis of connections between system components, such as metabolites. Correlation coefficients can be used to estimate how closely the metabolites are related to each other (Kose et al., 2001; Steuer, 2006; Jahagirdar and Saccenti, 2020). To visualize and analyze the correlation pattern, graph construction is widely used, where the metabolites are nodes, and the edges are the correlation links between these elements (Weckwerth et al., 2004; Perez De Souza et al., 2020). The result of this representation is shown in Figure 4. Networks were characterized by a scale-free structure, which is typical for biological systems (Jeong et al., 2000). The obtained correlation networks are distinguished by the presence of regions enriched with compounds of certain classes and connected metabolically, a similar pattern was observed in other systems (Fukushima et al., 2011; Shtark et al., 2021).

Apparently, the structure of the networks differs significantly. Correlation patterns have been shown as specific to the genotype, including point mutations (Fukushima et al., 2011), and to environmental conditions (Szymanski et al., 2009; Kotze et al., 2013). This is the result of the fact that external and internal factors cause changes in the state of the system, including rearrangements of the connections between metabolites, which is reflected in their correlation patterns (Camacho et al., 2005; Steuer, 2006; Rosato et al., 2018). Among the global differences between the networks, it is important to mention the maximum diameter, the characteristic path, and the heterogeneity of the network in heterotrophy. This indicates a decrease in the connecti- 
vity of the network, that is, the breakdown of metabolic connections. The reason for this effect may be the simplification of metabolism as a result of narrowing the range of available sources of energy and carbon to one-acetate.

\section{Conclusions}

The source of carbon under mixotrophic conditions is primarily acetate; so, blocking the fixation of $\mathrm{CO}_{2}$ has a weak effect on the growth rate and represses only autotrophic accumulation of biomass after acetate depletion. At the same time, light plays the role of the main source of energy and placing the culture in the dark leads to a drastic decrease in the growth rate. The dysfunction of the Calvin cycle leads to a complex of molecular and biochemical changes that contribute to the rerouting of carbon of acetate from the TCA into gluconeogenesis through a glyoxylate shunt. This occurs together with a decrease in the activity of the flow through the oxidative part of the TCA. Together with the decrease in the plastid transport of dicarboxylates, this leads to the suppression of nitrogen metabolism and the synthesis of amino acids. Besides, a decrease in the activity of TCA, apparently, causes an increase in the synthesis of lipids under illumination. Failure of the Calvin cycle also leads to a decrease in the activity of direct ATP transport into the plastid under illumination. Alternatively, in the dark, direct energy transport is maximized. As a result, we see an amazing systemic multi-level picture of the cell behavior, which is using flexibly its limited capabilities to adapt to capriciously changing circumstances.

\section{Acknowledgements}

This work was supported by the project of BIN RAS \#AAAA-A18-118032390136-5. We thank the Research Resource Center for Molecular and Cell Technologies (RRC MCT) of St. Petersburg State University for equipment access and technical support.

\section{References}

Arbona V., Manzi M., Ollas C. and Gymez-Cadenas A. 2013. Metabolomics as a tool to investigate abiotic stress tolerance in plants. Int. J. Mol. Sci. 14, 4885-4911.
Atteia A., Adrait A., Brugiere S., Tardif M., Lis R., Deusch O., Dagan T., Kuhn L., Gontero B., Martin W., Garin J., Joyard J. and Rolland N. 2009. A proteomic survey of Chlamydomonas reinhardtii mitochondria sheds new light on the metabolic plasticity of the organelle and on the nature of the proteobacterial mitochondrial ancestor. Mol. Biol. Evol. 26, 1533-1548.

Ball S. G. 1998. Regulation of starch biosynthesis. In: The molecular biology of chloroplasts and mitochondria in Chlamydomonas. Advances in photosynthesis. (Eds: Rochaix J. D., GoldschmidtClermont M. and Merchant S.). Kluwer Academ. Publ., Dordrecht, Netherlands. Vol 7, pp. 550-567.

Belcher J.H. 1968. Notes on the physiology of Botryococcus braunii Kützing. Arch. Mikrobiol. 61, 335-346.

Bogaert K.A., Perez E., Rumin J., Giltay A., Carone M., Coosemans N., Radoux M., Eppe G., Levine R.D., Remacle F. and Remacle C. 2019. Metabolic, physiological, and transcriptomics analysis of batch cultures of the green microalga Chlamydomonas grown on different acetate concentrations. Cells. 8, 1367.

Bölling C. and Fiehn O. 2005. Metabolite profiling of Chlamydomonas reinhardtii under nutrient deprivation. Plant Physiol. 139, 1995-2005.

Boschetti A. and Schmid K. 1998. Energy supply for atp-synthase deficient chloroplasts of Chlamydomonas reinhardii. Plant Cell Physiol. 39, 160-168.

Boyle N. R., Sengupta N. and Morgan J. A. 2017. Metabolic flux analysis of heterotrophic growth in Chlamydomonas reinhardtii. PLOS ONE. 12, e0177292.

Bracher A., Whitney S.M., Hartl F.U. and Hayer-Hartl M. 2017. Biogenesis and metabolic maintenance of Rubisco. Ann. Rev.Plant Biol. 68, 29-60.

Breuers R.K.H. 2011. The plastid outer envelope - a highly dynamic interface between plastid and cytoplasm. Front. Plant Sci. 2, 97.

Bulté L., Gans P., Rebéillé F. and Wollman F. A. 1990. ATP control on state transitions in vivo in Chlamydomonas reinhardtii. Biochim. Biophys. Acta (BBA) - Bioenergetics. 1020, 72-80.

Camacho D., de la A., Fuente A. and Mendes P. 2005. The origin of correlations in metabolomics data. Metabolomics. 1, 53-63.

Cardol P., Gloire G., Havaux M., Remacle C., Matagne R. and Franck F. 2003. Photosynthesis and state transitions in mitochondrial mutants of Chlamydomonas reinhardtii affected in respiration. Plant Physiol. 133, 2010-2020. 
Castro-Moretti F. R., Gentzel I. N., Mackey D. and Alonso A. P. 2020. Metabolomics as an emerging tool for the study of plant - pathogen interactions. Metabolites. 10, 52.

Cross F.R. and Umen J.G. 2015. The Chlamydomonas cell cycle. The Plant J. 82, 370-392.

Deng X., Cai J. and Fei X. 2013. Effect of the expression and knockdown of citrate synthase gene on carbon flux during triacylglycerol biosynthesis by green algae Chlamydomonas reinhardtii. BMC Biochem. 14, 38.

Eisenhut M., Hocken N. and Weber A. P. 2015. Plastidial metabolite transporters integrate photorespiration with carbon, nitrogen, and sulfur metabolism. Cell Calcium. 58, 98-104.

Eppley R.W., Gee R. and Saltman P. 1963. Photometabolism of acetate by Chlamydomonas mundana. Physiol. Plant. 164, 777-792.

Ermilova E.V., Chekunova E.M., Zalutskaya Z.M., Krupnov K.R. and Gromov B.V. 1996. Isolation and characterization of chemotaxis mutants of Chlamydomonas reinhardtii. Curr. Microbiol. 32, 357-359.

Ermilova E.V., Zalutskaya Z.M. and Gromov B.V. 1993. Chemotaxis towards sugars in Chlamydomonas reinhardtii. Curr. Microbiol. 27, 47-50.

Ermilova E. V., Zalutskaya Zh. M., Lapina T. V., Nikitin M. M. and Gromov B. V. 2000a. Flagellum functioning in the control of Chlamydomonas reinhardtii tactic movements. Rus. J.Plant Physiol. 47, 660-663.

Ermilova E.V., Zalutskaya Z.M., Gromov B.V., Häder D. and Purton S. 2000b. Isolation and characterisation of chemotactic mutants of Chlamydomonas reinhardtii obtained by insertional mutagenesis. Protist. 151, 127-137.

Facchinelli F. and Weber A. P. M. 2011. The metabolite transporters of the plastid envelope: an update. Front. Plant Sci. 2, 50.

Fang W., Si Y., Douglass S., Casero D., Merchant S. S., Pellegrini M., Ladunga I., Liu P. and Spalding M. H. 2012. Transcriptome-wide changes in Chlamydomonas reinhardtii gene expression regulated by carbon dioxide and the $\mathrm{CO}_{2}$-concentrating mechanism regulator CIA5/CCM1. Plant Cell. 24, 1876-1893.

Fett J.P. and Coleman J.R. 1994. Regulation of periplasmic carbonic anhydrase expression in Chlamydomonas reinhardtii by acetate and ph. Plant Physiol. 106, 103-108.

Fischer B.B., Wiesendanger M. and Eggen R.I.L. 2006. Growth condition-dependent sensitivity, photodamage and stress response of Chlamydomonas reinhardtii exposed to high light conditions. Plant Cell Physiol. 47, 1135-1145.

Flugge U., Hausler R. E., Ludewig F. and Gierth M. 2011. The role of transporters in supplying energy to plant plastids. J. Exp. Bot. 62, 2381-2392.

Fukushima A., Kusano M., Redestig H., Arita M. and Saito K. 2011. Metabolomic correlationnetwork modules in Arabidopsis based on a graphclustering approach. BMC Syst. Biol. 5, 1.

Gans P. and Rebeille F. 1990. Control in the dark of the plastoquinone redox state by mitochondrial activity in Chlamydomonas reinhardtii. Biochim. Biophys. Acta (BBA) - Bioenergetics. 1015, 150-155.

Gans P. and Wollman F. 1995. The effect of cyanide on state transitions in Chlamydomonas reinhardtii. Biochimica et Biophysica Acta (BBA). Bioenergetics. 1228, 51-57.

Geigenberger P., Stamme C., Tjaden J., Schulz A., Quick P.W., Betsche T., Kersting H.J. and Neuhaus H.E. 2001. Tuber physiology and properties of starch from tubers of transgenic potato plants with altered plastidic adenylate transporter activity. Plant Physiol. 125, 1667-1678.

Goodenough U., Blaby I., Casero D., Gallaher S.D., Goodson C., Johnson S., Lee J., Merchant S.S., Pellegrini M., Roth R., Rusch J., Singh M., Umen J.G., Weiss T.L. and Wulan T. 2014. The path to triacylglyceride obesity in the sta6 strain of Chlamydomonas reinhardtii. Eukaryot. Cell. 13, 591-613.

Goodson C., Roth R., Wang Z. T. and Goodenough U. 2011. Structural correlates of cytoplas-mic and chloroplast lipid body synthesis in Chlamydomonas reinhardtii and stimulation of lipid body production with acetate boost. Eukaryot. Cell. 10, 1592-1606.

Gorman D. and Levine R. 1965. Cytochrome f and plastocyanin: their sequence in the photosynthetic electron transport chain of Chlamydomonas reinhardii. PNAS 54, 1665-1669.

Goulding K.H., and Merrett M.J. 1966. The photometabolism of acetate by Chlorella pyrenoidosa. J. Exp. Bot. 17, 678-689.

Goulding K.H., and Merrett M.J. 1967. The photo-assimilation of acetate by Pyrobotrys (Chlamydobotrys) stellate. J. Gen. Microbiol. 48, 127-136.

Grossman A. 2000. Acclimation of Chlamydomonas reinhardtii to its nutrient environment. Protist. 151, 201-224.

Gu Z., Eils R. and Schlesner M. 2016. Complex heatmaps reveal patterns and correlations in multidimensional genomic data. Bioinformatics. 32, $2847-2849$. 
Gurrieri L., Fermani S., Zaffagnini M., Sparla F. and Trost P. 2021. Calvin $\backslash$ Benson cycle regulation is getting complex. Trends Plant Sci. 26, 898-912.

Haferkamp I., Fernie A.R. and Neuhaus H.E. 2011. Adenine nucleotide transport in plants: much more than a mitochondrial issue. Trends Plant Sci. 16, 507-515.

Harrop L.C. and Kornberg H.L. 1966. The role of isocitrate lyase in the metabolism of algae. Proc. R. Soc. London, Ser. B. Biol. Sci. 166, 11-29.

Hastie T., Tibshirani R., Narasimhan B. and Chu G. 2019. Impute: imputation for microarray data. R Package Version 1.60. Available online: https://bioconductor.org/packages/release/bioc/ html/impute.html.

Hayashi Y., Sato N., Shinozaki A. and Watanabe M. 2015. Increase in peroxisome number and the gene expression of putative glyoxysomal enzymes in Chlamydomonas cells supplemented with acetate. J. Plant Res. 128, 177-185.

Heifetz P.B., Förster B., Osmond C.B., Giles L.J. and Boynton J.E. 2000. Effects of acetate on facultative autotrophy in Chlamydomonas reinhardtii assessed by photosynthetic measurements and stable isotope analyses. Plant Physiol. 122, 1439-1446.

Heldt H. W. 1969. Adenine nucleotide translocation in spinach chloroplasts. FEBS Lett. 5, 11 -14.

Heldt H. W. and Rapley L. 1970. Specific transport of inorganic phosphate, 3-phosphoglycerate and dihydroxyacetonephosphate, and of dicarboxylates across the inner membrane of spinach chloroplasts. FEBS Lett. 10, 143-148.

Hudock G.A. and Levine R.P. 1964. Regulation of photosynthesis in Chlamydomonas reinhardtii. Plant Physiol. 39, 889-897.

Igamberdiev A.U. and Bykova N.V. 2018. Role of organic acids in the integration of cellular redox metabolism and mediation of redox signalling in photosynthetic tissues of higher plants. Free Rad. Biol. Med. 122, 74-85.

Igamberdiev A.U. and Eprintsev A.T. 2016. Organic acids: the pools of fixed carbon involved in redox regulation and energy balance in higher plants. Front. Plant Sci. 7, 1042.

Jahagirdar S. and Saccenti E. 2020. On the use of correlation and $\mathrm{mi}$ as a measure of metabolite -metabolite association for network differential connectivity analysis. Metabolites. 10, 171.

Jeong H., Tombor B., Albert R., Oltvai Z.N. and Barabбsi A. 2000. The large-scale organization of metabolic networks. Nature. 407, 651-654.

Jiang Y., Xiao P., Shao Q., Qin H., Hu Z., Lei A. and Wang J. 2017. Metabolic responses to ethanol and butanol in Chlamydomonas reinhardtii. Biotech. Biofuels. 10, 1.

Johnsen L.G., Skou P.B., Khakimov B. and Bro R. 2017. Gas chromatography - mass spectrometry data processing made easy. J. Chromatography A. 1503, 57-64.

Johnson X. and Alric J. 2013. Central carbon metabolism and electron transport in Chlamydomonas reinhardtii: metabolic constraints for carbon partitioning between oil and starch. Eukar. Cell. 12, 776-793.

Kajikawa M. and Fukuzawa H. 2020. Algal autophagy is necessary for the regulation of carbon metabolism under nutrient deficiency. Front. Plant Sci. 11, 36.

Kinoshita H., Nagasaki J., Yoshikawa N., Yamamoto A., Takito S., Kawasaki M., Sugiyama T., Miyake H., Weber A. P. M. and Taniguchi M. 2011. The chloroplastic 2-oxoglutarate/malate transporter has dual function as the malate valve and in carbon/nitrogen metabolism. Plant J. 65, 15-26.

Klein U., Chen C. and Gibbs M. 1983. Photosynthetic properties of chloroplasts from Chlamydomonas reinhardii. Plant Physiol. 72, 488-491.

Klöck G. and Kreuzberg K. 1991. Compartmented metabolite pools in protoplasts from the green alga Chlamydomonas reinhardtii: changes after transition from aerobiosis to anaerobiosis in the dark. Biochim. Biophys. Acta (BBA) - Gen. Subj. 1073, 410-415.

Kose F., Weckwerth W., Linke T. and Fiehn O. 2001. Visualizing plant metabolomic correlation networks using clique-metabolite matrices. Bioinformatics. 17, 1198-1208.

Kotze H.L., Armitage E.G., Sharkey K.J., Allwood J.W., Dunn W.B., Williams K.J. and Goodacre R. 2013. A novel untargeted metabolomics correlation-based network analysis incorporating human metabolic reconstructions. BMC Syst. Biol. 7, 107.

Krapp A., Hofmann B., Schafer C. and Stitt M. 1993. Regulation of the expression of RbcS and other photosynthetic genes by carbohydrates: a mechanism for the 'sink regulation' of photosynthesis? Plant J. 3, 817-828.

Lauersen K. J., Willamme R., Coosemans N., Joris M., Kruse O. and Remacle C. 2016. Peroxisomal microbodies are at the crossroads of acetate assimilation in the green microalga Chlamydomonas reinhardtii. Algal Research. 16, 266-274.

Lee D.Y., Park J., Barupal D.K. and Fiehn O. 2012. System response of metabolic networks in Chlamydomonas reinhardtii to total available 
ammonium. Mol. Cell. Proteomics. 11, 973-988.

Lee D. and Fiehn O. 2008. High quality metabolomic data for Chlamydomonas reinhardtii. Plant Meth. 4, 7.

Lemaire C., Wollman F.A. and Bennoun P. 1988. Restoration of phototrophic growth in a mutant of Chlamydomonas reinhardtii in which the chloroplast $\operatorname{atp} B$ gene of the ATP synthase has a deletion: an example of mitochondria-dependent photosynthesis. Proc. Natl. Acad. Sci. 85, 1344-1348.

Lemaire S. D., Guillon B., Le Marechal P., Keryer E., Miginiac-Maslow M. and Decottignies P. 2004. New thioredoxin targets in the unicellular photosynthetic eukaryote Chlamydomonas reinhardtii. Proc. Natl. Acad. Sci. 101, 7475-7480.

Liaw A. and Wiener M. 2002. Classification and regression by random forest. R. News. 2, 18-22.

Liu C., Wu G., Huang X., Liu S. and Cong B. 2012. Validation of housekeeping genes for gene expression studies in an ice alga Chlamydomonas during freezing acclimation. Extremophiles. 16, 419-425.

Livak K. J. and Schmittgen T. D. 2001. Analysis of relative gene expression data using real-time quantitative PCR and the $2^{-\triangle \Delta C T}$ method. Methods. 25, 402-408.

Lucker B. and Kramer D. M. 2013. Regulation of cyclic electron flow in Chlamydomonas reinhardtii under fluctuating carbon availability. Photosynth. Res. 117, 449-459.

Lv H., Qu G., Qi X., Lu L., Tian C. and Ma Y. 2013. Transcriptome analysis of Chlamydomonas reinhardtii during the process of lipid accumulation. Genomics. 101, 229-237.

Marchand J., Heydarizadeh P., Schoefs B. and Spetea C. 2018. Ion and metabolite transport in the chloroplast of algae: lessons from land plants. Cell. Mol. Life Sci. 75, 2153-2176.

Martínez-Rivas J. M. and Vega J. M. 1993. Effect of culture conditions on the isocitrate dehydrogenase and isocitrate lyase activities in Chlamydomonas reinhardtii. Physiol. Plant. 88, 599-603.

Matsuo T. and Ishiura M. 2010. New insights into the circadian clock in Chlamydomonas. Int.1 Rev. Cell Mol. Biol. 280, 281-314.

Mettler T., Mühlhaus T., Hemme D., Schöttler M.-A. et al. 2014. Systems analysis of the response of photosynthesis, metabolism, and growth to an increase in irradiance in the photosynthetic model organism Chlamydomonas reinhardtii. Plant Cell. 26, 2310-2350.
Moll B. and Levine R. P. 1970. Characterization of a photosynthetic mutant strain of Chlamydomonas reinhardii deficient in phosphoribulokinase activity. Plant Physiol. 46, 576-580.

Moon M., Kim C. W., Park W., Yoo G., Choi Y. and Yang J. 2013. Mixotrophic growth with acetate or volatile fatty acids maximizes growth and lipid production in Chlamydomonas reinhardtii. Algal Res. 2, 352-357.

Noctor G. and Foyer C.H. 2000. Homeostasis of adenylate status during photosynthesis in a fluctuating environment. J. Exp. Bot. 51, 347-356.

Nogales J., Guijo M.I., Quesada A. and Merch F. 2004. Functional analysis and regulation of the malate synthase from Chlamydomonas reinhardtii. Planta. 219, 325-331.

Noguchi K. and Yoshida K. 2008. Interaction between photosynthesis and respiration in illuminated leaves. Mitochondrion. 8, 87-99.

Ono K. and Watanabe A. 1997. Levels of endogenous sugars, transcripts of $r b c S$ and $r b c L$, and of $\mathrm{RuBisCO}$ protein in senescing sunflower leaves. Plant Cell Physiol. 38, 1032-1038.

Ostroukhova M. and Ermilova E. 2019. New insights into NO generation and AOX1 upregulation in Chlamydomonas. Protistology. 13, 19-25.

Oswald O., Martin T., Dominy P. J. and Graham I. A. 2001. Plastid redox state and sugars: interactive regulators of nuclear-encoded photosynthetic gene expression. Proc. Natl. Acad. Sci. 98, 2047-2052.

Perez De Souza L., Alseekh S., Brotman Y. and Fernie A. R. 2020. Network-based strategies in metabolomics data analysis and interpretation: from molecular networking to biological interpretation. Expert Review of Proteomics. 17, 243-255.

Pérez-Enciso M. and Tenenhaus M. 2003. Prediction of clinical outcome with microarray data: a partial least squares discriminant analysis (PLS-DA) approach. Hum. Genet. 12, 581-92.

Plancke C., Vigeolas H., Hohner R., Roberty S. et al. 2014. Lack of isocitrate lyase in Chlamydomonas leads to changes in carbon metabolism and in the response to oxidative stress under mixotrophic growth. Plant J. 77, 404-417.

Puzanskiy R., Tarakhovskaya E., Shavarda A. and Shishova M. 2018a. Metabolomic and physiological changes of Chlamydomonas reinhardtii (Chlorophyceae, chlorophyta) during batch culture development. J. Appl. Phycol. 30, 803-818.

Puzanskiy R., Romanyuk D. and Shishova M. 2018b. Coordinated alterations in gene expression 
and metabolomic profiles of Chlamydomonas reinhardtii during batch autotrophic culturing. Biol. Commun. 63, 87-99.

Puzanskiy R.K., Romanyuk D.A. and Shishova M.F. 2020a. Shift in expression of the genes of primary metabolism and chloroplast transporters in Chlamydomonas reinhardtii under different trophic conditions. Russ. J. Plant Physiol. 67, 867-878.

Puzanskiy R.K., Romanyuk D.A., Kirpichnikova A.A. and Shishova M.F. 2020b. Alteration in the expression of genes encoding primary metabolism enzymes and plastid transporters during the culture growth of Chlamydomonas reinhardtii. Mol. Biol. 54, 503-519.

Puzanskiy R., Shavarda A., Romanyuk D. and Shishova M. 2021. The role of trophic conditions in the regulation of physiology and metabolism of Chlamydomonas reinhardtii during batch culturing. J. Appl. Phycol. 33, 2897-2908.

Reinhold T., Alawady A., Grimm B., Beran K.C., Jahns P., Conrath U., Bauer J., Reiser J., Melzer M., Jeblick W. and Neuhaus H.E. 2007. Limitation of nocturnal import of ATP into Arabidopsis chloroplasts leads to photooxidative damage: plastidial nucleotide transporter. Plant J. 50, 293 -304 .

Reiser J., Linka N., Lemke L., Jeblick W. and Neuhaus H.E. 2004. Molecular physiological analysis of the two plastidic atp/adp transporters from Arabidopsis. Plant Physiol. 136, 3524-3536.

Renberg L., Johansson A. I., Shutova T., Stenlund H., Aksmann A., Raven J. A., Gardeström P., Moritz T. and Samuelsson G. 2010. A metabolomic approach to study major metabolite changes during acclimation to limiting $\mathrm{CO}_{2}$ in Chlamydomonas reinhardtii. Plant Physiol. 154, 187-196.

Roach T., Sedoud A. and Krieger-Liszkay A. 2013. Acetate in mixotrophic growth medium affects photosystem II in Chlamydomonas reinhardtii and protects against photoinhibition. Biochim. Biophys. Acta (BBA) - Bioenergetics. 1827, 1183-1190.

Rochaix J. 2001. Assembly, function, and dynamics of the photosynthetic machinery in Chlamydomonas reinhardtii. Plant Physiol. 127, 1394-1398.

Roesler K. R., Marcotte B. L. and Ogren W. L. 1992. Functional importance of arginine 64 in Chlamydomonas reinhardtii phosphoribulokinase. Plant Physiol. 98, 1285-1289.

Rosato A., Tenori L., Cascante M. and De Atauri Carulla P. R., Martins dos Santos V. A. P., Saccenti E. 2018. From correlation to causation: analysis of metabolomics data using systems biology approaches. Metabolomics. 14, 37.
Sager R. and Granick S. 1953. Nutritional studies with Chlamydomonas reinhardii. Ann. N.Y. Acad. Sci. 56, 831-838.

Salomé P. A. and Merchant S. S. 2019. A series of fortunate events: introducing Chlamydomonas as a reference organism. Plant Cell. 31, 1682-1707.

Samuelsson G. and Oquist G. 1977. A method for studying photosynthetic capacities of unicellular algae based on in vivo chlorophyll fluorescence. Physiol. Plant. 40, 315-319.

Sanz-Luque E., Chamizo-Ampudia A., Llamas A., Galvan A. and Fernandez E. 2015. Understanding nitrate assimilation and its regulation in microalgae. Front. Plant Sci. 6, 899.

Scheibe R. 2004. Malate valves to balance cellular energy supply. Physiol. Plant. 120, 21-26.

Scheibe R., Backhausen J. E., Emmerlich V. and Holtgrefe S. 2005. Strategies to maintain redox homeostasis during photosynthesis under changing conditions. J. Exp. Bot. 56, 1481-1489.

Schneidereit J., Häusler R. E., Fiene G., Kaiser W.M. and Weber A.P. 2006. Antisense repression reveals a crucial role of the plastidic 2-oxoglutarate/ malate translocator DiT1 at the interface between carbon and nitrogen metabolism. Plant J. 45, 206 -224 .

Scranton M. A., Ostrand J. T., Fields F. J. and Mayfield S. P. 2015. Chlamydomonas as a model for biofuels and bioproducts production. Plant J. 82, 523-531.

Shannon P. 2003. Cytoscape: a software environment for integrated models of biomolecular interaction networks. Genome Res. 13, 2498-2504.

Shtark O., Puzanskiy R., Avdeeva G., Yemelyanov V., Shavarda A., Romanyuk D., Kliukova M., Kirpichnikova A., Tikhonovich I., Zhukov V. and Shishova M. 2021. Metabolic alterations in Pisum sativum roots during plant growth and arbuscular mycorrhiza development. Plants. 10, 1033.

Singh H., Shukla M.R., Chary K.V.R. and Rao B.J. 2014. Acetate and bicarbonate assimilation and metabolite formation in Chlamydomonas reinhardtii: a ${ }^{13} \mathrm{C}-N M R$ study. PLoS ONE. 9, e106457.

Stacklies W., Redestig H., Scholz M., Walther D. and Selbig J. 2007. pcaMethods - a bioconductor package providing PCA methods for incomplete data. Bioinformatics., 23, 1164-1167.

Steuer R. 2006. Review: on the analysis and interpretation of correlations in metabolomic data. Brief. Bioinform. 7, 151-158.

Szymanski J., Jozefczuk S., Nikoloski Z., Selbig J., Nikiforova V., Catchpole G. and Willmitzer L. 2009. Stability of metabolic correlations under 
changing environmental conditions in Escherichia coli - a systems approach. PLoS ONE. 4, e7441.

Taniguchi M., Taniguchi Y., Kawasaki M., Takeda S., Kato T., Sato S., Tabata S., Miyake H. and Sugiyama T. 2002. Identifying and characterizing plastidic 2-oxoglutarate/malate and dicarboxylate transporters in arabidopsis thaliana. Plant Cell Physiol. 43, 706-717.

Terashima M., Specht M., Naumann B. and Hippler M. 2010. Characterizing the anaerobic response of Chlamydomonas reinhardtii by quantitative proteomics. Mol. Cell. Prot. 9, 1514-1532.

Terauchi A.M., Peers G., Kobayashi M.C., Niyogi K.K. and Merchant S.S. 2010. Trophic status of Chlamydomonas reinhardtii influences the impact of iron deficiency on photosynthesis. Photosynth. Res. 105, 39-49.

Therien J.B., Zadvornyy O.A., Posewitz M.C., Bryant D.A. and Peters J.W. 2014. Growth of Chlamydomonas reinhardtii in acetate-free medium when co-cultured with alginate-encapsulated, acetateproducing strains of Synechococcus sp. PCC 7002. Biotech. Biofuels. 7, 154.

Tjaden J., Mohlmann T., Kampfenkel K., Henrichs G. and Neuhaus H.E. 1998. Altered plastidic ATP/ADP-transporter activity influences potato (Solanum tuberosum L.) tuber morphology, yield and composition of tuber starch. Plant J. 16, 531-540.

Togasaki R. K. and Levine R. P. 1970. Chloroplast structure and function in ac-20, a mutant strain of Chlamydomonas reinhardtii. J. Cell Biol. 44, 531-539.

Wang Y., Stessman D.J. and Spalding M.H. 2015. The $\mathrm{CO}_{2}$ concentrating mechanism and photosynthetic carbon assimilation in limiting $\mathrm{CO}_{2}$ : how Chlamydomonas works against the gradient. Plant J. 82, 429-448.

Weber A. P. and Linka N. 2011. Connecting the plastid: transporters of the plastid envelope and their role in linking plastidial with cytosolic metabolism. Ann. Rev. Plant Biol. 62, 53-77.

Weber A. and Flügge U. 2002. Interaction of cytosolic and plastidic nitrogen metabolism in plants. J. Exp. Bot. 53, 865-874.

Weckwerth W., Loureiro M. E., Wenzel K. and Fiehn O. 2004. Differential metabolic networks unravel the effects of silent plant phenotypes. Proc. Natl. Acad. Sci. 101, 7809-7814.

Wienkoop S., Weiß J., May P., Kempa S., Irgang S., Recuenco-Munoz L., Pietzke M., Schwemmer T., Rupprecht J., Egelhofer V. and Weckwerth W. 2010. Targeted proteomics for Chlamydomonas reinhardtii combined with rapid subcellular protein fractionation, metabolomics and metabolic flux analyses. Mol. BioSyst. 6, 1018.

Woessner J.P., Masson A., Harris E.H., Bennoun P., Gillham N.W. and Boynton J.E. 1984. Molecular and genetic analysis of the chloroplast ATPase of Chlamydomonas. Plant Mol. Biol. 3, 177-190.

Yun E.J., Zhang G., Atkinson C., Lane S., Liu J., Ort D. R. and Jin Y. 2021. Glycolate production by a Chlamydomonas reinhardtii mutant lacking carbon-concentrating mechanism. J. Biotech. 335, 39-46.

Zhekisheva M., Boussiba S., Khozinâ-Goldberg I., Zarka A. and Cohen Z. 2002. Accumulation of oleic acid in Haematococcus pluvialis (Chlorophyceae) under nitrogen starvation or high light is correlated with that of astaxanthin esters. J. Phycol. 38, 325-331.

Zhou Y., Schideman L., Park D., Stirbet A., Govindjee G., Rupassara S., Krehbiel J. and Seufferheld M. 2015. Characterization of a Chlamydomonas reinhardtii mutant strain with improved biomass production under low light and mixotrophic conditions. Algal Res. 11, 134-147.

Address for correspondence: Maria Shishova. Biological Faculty, St. Petersburg State University, Universitetskaya Emb. 7/9, 199034 St. Petersburg, Russia; e-mail: mshishova@mail.ru 